\title{
Time temperature indicators for monitoring environment parameters during transport and storage of perishables: A Review
}

\author{
Shetty J. Manjunath $凶$
}

Received: 16.08.2018

Revised: 05.10.2018

Accepted: 28.11.2018

\begin{abstract}
Time temperature indicators are devices which integrate the exposure to temperature over time by accumulating the effect of such exposures and exhibiting a change of colour (or other physical characteristic). TTI's are commonly used in food, pharmaceutical and medical products to indicate exposure to excessive temperature abuse. TTI's help in assure the cool chain of food products, they are expected to reduce the amount of food waste, as well as reducing the number of food borne illnesses. The mandatory regulations on pharmaceutical cold chain logistics by international and national associations like WHO are influencing the demand for TTI labels. Global time temperature indicator labels market is expected to witness rapid growth during the forecast period. This growth is expected to be primarily driven by growing concern about food wastage and its impacts on economy.
\end{abstract}

Key words: Temperature indicators, time temperature indicators (TTI), polymerization,

\section{Introduction}

Food is packaged for storage, preservation, and protection traditionally for a long time. These three are the basis functions of food packaging that are still required today for better maintenance of quality and handling of foods (Galic et al., 2011). A modern quality and safety assurance system should prevent contamination through the monitoring, recording, and controlling of critical parameters during a product's entire life cycle, which includes the post processing phase and extends over the time of use by the final consumer (Koutsoumanis et al., 2005). An important indicator for monitoring the quality of packaged food is temperature. Time/ temperature indicators or integrators (TTIs) are used as cost-effective and user-friendly devices to monitor, record, and translate the overall effect of temperature history on food quality in the chill chain down to a product unit level (Taoukis, 2001; Taoukis and Labuza, 2003; Giannakourou et al., 2005). Depending on the working principle, TTI systems are classified as chemical, physico chemical, or biological systems, with their integrated time-temperature dependent change being manifested as an irreversible visible color

\section{Author's Address}

Department of Postharvest Technology, College of Horticulture, Bengaluru, UHS (B), Karnataka E-mail.: jshettymanju@gmail.com development a movement toward a color change, or a mechanical change in consistency. In this context, this review helps for the better understanding of different types of TTI and their working principles.

\section{Time Temperature Indicators}

The temperature variations in a food product can lead to changes in product safety and quality. A time temperature integrator or indicator (TTI) can be defined as a simple, inexpensive device that can show an easily measurable, time-temperature dependent change that reflects the full or partial temperature history of a food product to which it is attached (Taoukis and Labuza, 1989). The TTIs presently available on the market have working mechanisms based on different principle. The principle of TTI operation is a mechanical, chemical, enzymatic or microbiological irreversible change, usually expressed as a visible response in the form of a mechanical deformation, colour development or colour movement (Taoukis, 2008).

For chemical or physical response, it is based on chemical reaction or physical change towards time and temperature, such as acid-base reaction, melting, polymerization, etc. While for biological response, it is based on the change in biological activity, such as microorganism, spores or enzymes 
towards time or temperature (Kuswandi et al., 2011).The rate of change is temperature dependent, increasing at higher temperatures similarly to the deteriorative reactions responsible for product quality deterioration. The visible response of the TTI thus cumulatively reflects the time-temperature history of the product it accompanies (Taoukis, 2008). TTIs must be easily activated and then exhibit a reproducible time- temperature dependent change which is easily measured. This change must be irreversible and ideally mimic or be easily correlated to the food's extent of deterioration and residual shelf-life. TTIs may be classified as either partial history or full history indicators, depending on their response mechanism (Selman, 1995).

TTIs can be divided into two categories based on their data storage condition:

- Partial history indicators-which do not respond unless some predetermined threshold temperature is exceeded, and it is intended to identify abusive temperature conditions and

- Full history indicator-which respond continuously to all temperatures.

TTIs may be classified into three categories (Taoukis and Labuza, 2003):

- Critical temperature indicators (CTI) show exposure above (or below) a reference temperature. Denaturation of an important protein above the critical temperature or growth of a pathogenic microorganism is other important cases where a CTI would be useful.

- Critical temperature/time integrators (CTTI) are useful in indicating breakdowns in the distribution chain and for products in which reactions, important to quality or safety, are initiated or occur at measurable rates above a critical temperature. Examples of such reactions are microbial growth or enzymatic activity that is inhibited below the critical temperature.

- Time temperature integrators or indicators (TTI) give a continuous, temperature dependent response throughout the product's history.

\section{Different TTI's Ruling Market \\ 1. 3M MonitorMark ${ }^{\circledR}$}

The 3M MonitorMark ${ }^{\circledR}$ (3M Co., St Paul, Minnesota) is diffusion-based indicator label and is on the color change of an oxidable chemical system controlled by temperature-dependent permeation through a film. The action is activated by a blue- dyed fatty acid ester diffusing along a wick. A viscoelastic material migrates into a diffusely light reflective porous matrix at a temperature dependent rate. The response rate and temperature dependence is controlled by the tag configuration, the diffusing polymer's concentration and its glass transition temperature and can be set at the desirable range (Ahvenainen, Hurme, 1997; Taoukis, 2008).

Monitor Mark $®$ has two versions, one intended for monitoring distribution, the threshold indicator for industry, and other intended for consumer information, the smart label (Kuswandi et al., 2011). Response of the indicator is measured by the progression of the blue dye along the track, and this is complete when all five windows are blue. An indicator tag labeled 51, for example, would indicate a response temperature (melt temperature) of $5{ }^{\circ} \mathrm{C}$ with a response time of 2 days. This response refers to the time taken to complete blue colour for all five windows at a constant $2{ }^{\circ} \mathrm{C}$ above the response temperature of the tag. Similarly, response times of 7 days and 14 days are available on tags, with response temperatures varying from $-17{ }^{\circ} \mathrm{C}$ to $+48^{\circ} \mathrm{C}$ (Selman, 1995; de Kruijf et al., 2002).

- The 3M MonitorMark® TTI is a partial history indicator the dimension of monitor mark is

1. $88 \times 19 \mathrm{~mm}$ rectangular cardboard containing

2. $28 \times 12 \mathrm{~mm}$ pad of blue dye with a carrier substance,

3. plastic slip-tab for isolating the dye,

4. $7 \mathrm{~mm}$ blotter paper wick,

5. And $88 \times 19 \mathrm{~mm}$ rectangular cardboard with five window cuts

- The bottom piece has a pressure sensitive adhesive backing.

- Removal of the slip-tab is needed to activate it. Before activation it is needed to be stored at temp not less than $-40^{\circ} \mathrm{C}$

- This indicator has a scale to indicate the length of accumulated exposure time above a predetermined temperature.

\section{How Monitor Mark works?}

Removal of slip-tab brings the pad (containing dye + carrier compound) and wick (blotter paper wick) into contact. The blue dye remaining within the pad until the carrier substance undergoes a phase change due to temperature exposure above a response temperature. Typical esters are used as 
carrier compound like octyle octanoate, dimethyle phthalate, and butyl stearate. Indicator response is measured by reading the distance the dye front has migrated past the indicator's window cuts

\section{Timestrips ${ }^{\circledR}$}

The Timestrips ${ }^{\circledR}$ (Timestrip UK Limited, UK) are smart labels for monitor how long a product has been open or how long it has been in use. Temperature monitoring at home is also very important for food safety. Timestrip ${ }^{\circledR}$ is a singleuse consumer activated smart-label for monitoring elapsed time on perishable products. It was designed to enable consumers to record time elapsed since activation of the label. This functionality is particularly suitable for packaging or labeling perishable products or products requiring regular maintenance or replacement (refrigerated and frozen products). It automatically monitors lapsed time, from 10 minutes to 12 months. The label is automatically activated when the consumer opens the packaging or it can be supplied as an external label that consumers can manually activate when they first use a product (Selman, 1995; Kuswandi et al., 2011).

\section{Fresh-Check®}

The Fresh-Check®TTI (Temptime Corp., Morris Plains, NJ, USA) is based on a solid state polymerization reaction, resulting in a highly coloured polymer. The response of the TTI is the colour change measurable as a decrease in reflectance (Taoukis, 2008). The indicator consists of a small circle of a polymer surrounded by a printed reference ring. The inside polymer circle darkens if the package has experienced unfavorable temperature exposures and the intensity of the color is measured and compared to the reference color scale on the label (de Kruijf et al., 2002). The faster the temperature increases, the faster the color changes occur in the polymer. Consumers are advised not to consume or purchase the product, regardless of the "use-by" date (Han et al., 2005).This indicator may be applied to packages of perishable products to ensure consumers at pointof-purchase and at home that the product is still fresh. These indicators have been used on fruit cake, lettuce, milk, chilled food.

\section{CheckPoint $\AA$ / VITSAB}

The CheckPoint ${ }^{\circledR T T I}$ (VITSAB A. B., Malmö, Sweden) is a simple adhesive label on enzymatic system. The VITSAB $^{\mathrm{TM}}$ (Visual Indicator Tag System AB) time-temperature monitor is a full history indicator consisting of an inner transparent pouch with two compartments and an outer rectangular casing $(62 \times 25 \mathrm{~mm})$. These labels react to time and temperature in the same way that food product react, and thus give a signal about the state of freshness and remaining shelf-life. The TTI is based on a colour change caused by a $\mathrm{pH}$ decrease that is the result of a controlled enzymatic hydrolysis of a lipid substrate. Hydrolysis of the substrate causes acid release and the $\mathrm{pH}$ drop is translated into a colour change of a $\mathrm{pH}$ indicator from deep green to bright yellow to orange red.

\section{How VITSAB works}

VITSAB ${ }^{\circledR}$ is based on the color change caused by ${ }_{\mathrm{p}} \mathrm{H}$ reduction due to a controlled enzymatic hydrolysis of lipid substrate. The indicator is made of two distinct compartments, one containing an aqueous solution of lipolytic enzymes and the other mainly containing triglycerides and a $\mathrm{pH}$ indicator. The TTI activation is through a wall break between the two compartments and might be carried out manually or through in-line automation. The substrate hydrolysis reduces $\mathrm{pH}$ and results in color change (from dark green to bright yellow) on the $\mathrm{pH}$ screen. CheckPoint ${ }^{\circledR}$ labels are the latest labels designed by VITSB $\AA$ in order to provide a better response for consumers and offer direct application to poultry and ground beef products.

Different combinations of enzyme-substrate types and concentrations can be used to give a variety of response lives and temperature dependencies (Taoukis, 2008). The sequential development of colour is appropriate for signposts in the management of the self-life of the product (Kuswandi et al., 2011).

Types of VITSAB

- Master Carton Version - Designed as an early warning indicator, is applied to the cartons or pallets in factory, and deliberately activated by the pressure of the labeling machine. The colour change are as follows :

1) Green $\rightarrow$ Excellent quality (80\% or less of the product's time-temp tolerance is used up)

2) Yellow $\rightarrow$ Good quality ( $80 \%$ is used up) 
3) Brown -> Uncertain quality (100\% is used up)

4) Red $\rightarrow$ Overexposed quality (130\% or more is used up)

- Consumer Version - The consumer time-temp monitor is designed to place on individual consumer packages and consists of a single ampoule, which is activated at the time of packaging. Its function is to show two colour signals :

1) Green -> Fit for consumption

2) Yellow $\rightarrow$ Not fit for consumption

\section{OnVu ${ }^{\mathrm{TM}}$ TTI}

The $\mathrm{OnVu}^{\mathrm{TM}}$ TTI (Ciba Specialty Chemicals \& Freshpoint, SW) is a newly introduced solid state reaction TTI. It is based on photosensitive compounds; organic pigments e.g. benzylpyridines, that change colour with time at rates determined by temperature. The TTI labels consist of a heart shaped 'apple' motif containing an inner heart shape. The image is stable until activated by UV light from an LED lamp, when the inner heart changes to a deep blue colour. A filter is then added over the label to prevent it being recharged. The blue inner heart changes to white as a function of time and temperature. The system can be applied as a label or printed directly onto the package (Pocas, 2001; Taoukis, 2008; Tsironi et al., 2008).

\section{6. $(\mathrm{eO})(\mathrm{TTI}$}

The $(\mathrm{eO}){ }^{\circledR}$ TTI (CRYOLOG, Gentilly, France) is based on a time-temperature depended $\mathrm{pH}$ change caused by controlled microbial growth selected strains of lactic acid bacteria that is expressed to colour change through suitable $\mathrm{pH}$ indicators. Prior to utilization, these TTIs are stored in a frozen state $\left(-18^{\circ} \mathrm{C}\right)$ to prevent the bacterial growth in the TTI medium. As they are very thin, their activation is obtained simply by defrosting them for a few minutes at room temperature. Once they are put on the food, and in case of temperature abuse, or when the product reaches its use by date, the temperaturedependent growth of the TTI microorganisms causes a $\mathrm{pH}$ drop in the tags leading to an irreversible color change of the medium chromatic indicator which becomes red (Ellouze et al., 2008; Taoukis, 2008). Ellouze and Augustin (2010) evaluated $(\mathrm{eO}){ }^{\circledR}$, a biological TTI as a quality and safety indicator for ground beef and spiced cooked chicken slices packed under modified atmosphere.

\section{TEMPTIME ${ }^{\mathrm{TM}}$ Fresh-Scan $^{\circledR}$}

The TEMPTIME ${ }^{\mathrm{TM}}$ like Fresh-Scan ${ }^{\circledR}$ labels provide a full history TTI, showing a response independently of a temperature threshold.

The indicator consists of three distinctive regions:

a. An eight- digit no. unique to each indicator

b. A two-digit code that identifies the indicator model

c. A strip of material known as the indication band that changes colour as a result of accumulated temperature exposure.

The indicators have no means for in-field activation, and are shipped from the manufacturer already activated and responding to the storage temperature. To minimize indicator response prior to use, they are stored at $-24^{0} \mathrm{C}$.

\section{How TEMPTIME (Fresh-Scan) works}

The indication band contains diacetylene monomers, which appear colourless because they absorb light only in UV portion of the spectrum. They undergo time-temp dependent polymerization to form a polymer with a conjugated backbone on which electrons are delocalized. The delocalized electrons absorb light in the visible portion of the spectrum and the polymer appears coloured. A change of the side group causes a dramatic change in the solid-state reaction kinetics. Generally follows Arrhenius-type kinetics over a wide range of temp range. The colour change and the bar codes are monitored using specially programmed, handheld microcomputer with an optical wand, which records the decrease in reflection as the indication band darkens. The product shelf life can be calculated from the change in colour, with respect to the prior time-temp experiences fed to the program.

\section{TT Sensor Plus ${ }^{\mathrm{TM}}$}

TT Sensor Plus records the temperatures an item is exposed to throughout its supply chain journey. Based on the temperatures the product is exposed to, TT Sensor Plus can indicate if the item is suitable for its intended use. The data is stored in a chip and can be uploaded to a smartphone at anytime, anywhere throughout the supply chain. The technology incorporates sensor functionality and temperature data logging capabilities in a costeffective, disposable temperature data label solution. When affixed to a product or container, 
the thin, flexible label can be programmed, by the user, to record the temperature history of goods at defined intervals during shipment.

\section{Benefits of TTI}

- Low cost and accurate time-temperature data logger

- Small credit card-sized label with easy set up

- Mobile app programmable logging and wireless data transfer

- Lower shrinkages and greater sustainability

- Provides data to help improve shipment quality, inventory and logistic

\section{Applications of TTI}

- Food and beverages

- Pharmaceutical and medical industries

- Other temperature-sensitive goods

- Chemicals and polymers

\section{Key Features of TTI}

- Operating temperature range of $-20^{\circ} \mathrm{C}$ to $50^{\circ} \mathrm{C}$

- Embedded (NFC) and temperature sensor microchip

- Real-time clock for data logging

- Temperature alarm

- Temperature accuracy of $\pm 0.5^{\circ} \mathrm{C}$

- Temperature resolution $=.01^{\circ} \mathrm{C}$

- Corresponding mobile app to configure, read and manage temperature data

\section{Recent Developments in TTI's}

- Many new types of TTIs have recently been developed. For example, Wanihsuksombat et al., (2010) developed prototype of a lactic acid based time- temperature indicator for monitoring food quality.

- Galagan and Su (2008) developed a novel colorimetric TTI based on fadable ink.

- Vaikousi et al., (2009) developed a new TTI system based on the growth and metabolic activity of a Lactobacillus sakei strain for monitoring food quality throughout the chilled foodchain.

- Yan et al., (2003) developed a new amylasetype TTI based on the reaction between amylase and starch.

\section{Conclusion}

Use of time temperature indicators can help optimize product distribution, improve shelf life, monitoring and management of food produce and thus reduce product waste from foodstuffs. Cost, reliability, and effective application are criteria for practical success of time temperature indicators. Current time temperature indicators systems provide reliable and reproducible responses according to their specifications. Time-temperature indicators provide a visual summary about temperatures of product accumulated in time history, recording the effects of time and temperature. A modern quality and safety assurance system should prevent contamination through the monitoring, recording, and controlling of critical parameters such as temperature during a food product's entire life cycle. It includes the postprocessing phase and extends to the time of use by the consumer. Hence, monitoring and recording the temperature conditions during distribution and storage are of importance.

\section{References}

Abraham, S.P., Chuah, M.C., Sampath, A, Saraydar, C.U., 2006. Rate control system and method for a link within a wireless communications system. United States patent US inventors; Lucent Technologies Inc, assignee 7,031,254.

Ellouze, M. and Augustin, J.C., 2010. Applicability of biological time temperature integrators as quality and safety indicators for meat products. International journal of food microbiology, 138(1-2):119-129.

Ellouze, M., Pichaud, M., Bonaiti, C., Coroller, L., Couvert, O., Thuault, D. and Vaillant, R., 2008. Modelling $\mathrm{pH}$ evolution and lactic acid production in the growth medium of a lactic acid bacterium: application to set a biological TTI. International journal of food microbiology, 128(1):101-107.

Galagan, Y. and Su, W.F., 2008. Fadable ink for timetemperature control of food freshness: Novel new timetemperature indicator. Food research international, 41(6):653-657.

Galić, K., Ščetar, M. and Kurek, M., 2011. The benefits of processing and packaging. Trends in Food Science \& Technology, 22(2-3):127-137.

Giannakourou, M.C., Koutsoumanis, K., Nychas, G.J.E. and Taoukis, P.S., 2005. Field evaluation of the application of time temperature integrators for monitoring fish quality in the chill chain. International journal of food microbiology, 102(3):323-336. 


\section{Shetty J. Manjunath}

Han, J.H., Ho, C.H. and Rodrigues, E.T., 2005. Intelligent packaging. Innovations in food packaging :138-155.

Koutsoumanis, K., Taoukis, P.S. and Nychas, G.J.E., 2005. Development of a safety monitoring and assurance system for chilled food products. International journal of food microbiology, 100(1-3):253-260.

Kuswandi, B., Wicaksono, Y., Abdullah, A., Heng, L.Y. and Ahmad, M., 2011. Smart packaging: sensors for monitoring of food quality and safety. Sensing and Instrumentation for Food Quality and Safety, 5(3-4):137-146.

Pocas, M., 2001. Innovations in intelligent packaging technologies for perishable foods. Novel Processes and Control Technologies in the Food Industry, ed. by Bozoblu F, Deak T, Ray B. NATO-ASI, IOS press, Amsterdam:197-211.

Selman, J.D., 1995. Time-temperature indicators. In Active food packaging Springer, Boston, MA.:215-237.

Taoukis, P.S., 2001. Modelling the use of time-temperature indicators in distribution and stock rotation. Food process modelling:512.

Taoukis, P.S. and Labuza, T.P., 1989. Reliability of timetemperature indicators as food quality monitors under nonisothermal conditions. Journal of

Food
Tsironi, T., Gogou, E., Velliou, E. and Taoukis, P.S., 2008. Application and validation of the TTI based chill chain management system SMAS (Safety Monitoring and Assurance System) on shelf life optimization of vacuum packed chilled tuna. International Journal of Food Microbiology, 128(1):108-115.

Taoukis, P., Tsironi, T., Gogou, E. and Giannoglou, M., 2008, June. Chill Chain Management and Shelf Life Optimisation of MAP Seabream Fillets: A TTI Based Alternative to FIFO. In 3rd International Workshop Cold Chain Management:2-3.

Vaikousi, H., Biliaderis, C.G. and Koutsoumanis, K.P., 2009. Applicability of a microbial time temperature indicator (TTI) for monitoring spoilage of modified atmosphere packed minced meat. International Journal of Food Microbiology, 133(3):.272-278.

Wanihsuksombat, C., Hongtrakul, V. and Suppakul, P., 2010 Development and characterization of a prototype of a lactic acid-based time-temperature indicator for monitoring food product quality. Journal of Food Engineering, 100(3):427-434.

Yan, S., Huawei, C., Limin, Z., Fazheng, R., Luda, Z. and Hengtao, Z., 2008. Development and characterization of a new amylase type time-temperature indicator. Food control, 19(3):315-319. 